\title{
The Mind Machine
}

\author{
Dr Richard Wiseman \& Emma Greening \\ Perrott-Warrick Research Unit \\ Psychology Department \\ University of Hertfordshire \\ College Lane \\ Hatfield \\ Herts \\ AL10 9AB
}

\begin{abstract}
For many years scientists have examined the possible existence of extra-sensory perception (ESP). One of the most common types of experiment, referred to as a 'forced choice' study, involves participants attempting to guess the identity of hidden targets that have been randomly selected from a set of alternatives known to participants prior to making their guess. Many researchers have argued that the results of these experiments provide strong support for the existence of psychic ability. However, others have criticised many of the experiments on both methodological and statistical grounds. The authors aimed to help resolve this debate by devising a novel way of carrying out a large scale forced choice ESP experiment. The Mind Machine consisted of a specially designed steel cabinet containing a multi-media computer and large touch screen monitor. The computer presented participants with a series of videoclips that led them through the experiment. During the experiment participants were asked to complete a forced choice ESP task that involved them guessing the outcome of four random electronic coin tosses. All of their data was stored by the computer during an eleven month tour of some of Britain's largest shopping centres, museums and science festivals. 27,856 participants contributed 110,959 trials, and thus the final database had the statistical power to detect the possible existence of a very small ESP effect. However, the cumulated outcome of the trials was consistent with chance. The experiment also examined the possible relationship between participants' ESP scores and their gender, belief in psychic ability and degree of predicted success. The results from all of these analyses were non-significant. Also, scoring on 'clairvoyance' trials (where the target was selected prior to the participant's choice) was not significantly different from 'precognitive' trials (where the target was chosen after the participants had made their choice). Competing interpretations of these findings are discussed, along with suggestions for future research.
\end{abstract}

\section{Acknowledgments}

The authors would like to thank The Committee On The Public Understanding of Science, The Perrott Warrick Fund, Geomica and Barcrest for supporting the work described in this paper. We also wish to acknowledge the assistance provided by John Bain, Adrian Owen, Tina Gutbrod, Caroline Watt, Mike Hutchinson, Susan Blackmore and the staff at each of the venues who were kind enough to host the Mind Machine. 


\section{Introduction}

Parapsychologists have carried out a large number of studies examining the possible existence of extra-sensory perception (ESP). One of the most principal types of experimental design uses the 'forced choice' procedure, in which participants are asked to guess the identity of hidden 'targets' (e.g., the colour of playing cards) that have been randomly selected from a set of alternatives known to participants prior to making their guess (e.g., they are told that the cards will be either red or black).

Many of the early forced choice ESP experiments were conducted by Rhine and his colleagues at Duke University in the early part of the last century (see Pratt, Rhine, Smith, Stuart \& Greenwood, 1940/1966). The majority of this work involved participants attempting to guess the order of shuffled packs of cards carrying the image of a star, circle, square, cross or wavy lines. These studies were often very labour intensive, and involved data collection and analysis being carried out by hand. Recent research has tended to use more automated procedures. For example, Schmidt (1969) developed an electronic device that randomly selected one of four lamps, prompted participants to indicate which lamp they thought the device had selected and provided feedback by lighting the target lamp after they had registered their choice. Similarly, Honorton (1987) developed 'ESPerciser' - a computerbased system that presented participants with four on-screen boxes and asked them to guess which one had been randomly selected by the computer. Both systems automatically recorded information about both the selected targets and participant choices.

These experiments have investigated a wide range of hypotheses (see Palmer, 1978, for a review). Some studies have examined the possible existence of telepathy by having another person, referred to as a 'sender', concentrate on targets prior to the participant's guess. Other work has investigated clairvoyance by having participants attempt to guess the identity of targets that are not known to anyone else (e.g., the order of a deck of cards that have been shuffled and immediately sealed in an envelope). A third set of studies has examined participants' precognitive abilities by, for example, having them predict the order of a deck of cards, shuffling the cards and then comparing the predicted order with the actual order. These studies have also examined how ESP scores are affected by different kinds of target material (e.g., symbols vs words), experimental procedures (e.g., providing feedback to participants about their scores vs not providing feedback) and individual differences (e.g., those that believe in ESP vs disbelievers).

Many researchers have argued that the results of these studies support the existence of ESP. For example, Pratt et al. (1940/1966) reviewed the findings from more than 3.6 million guesses made in over 140 forced choice ESP studies conducted between 1882 and 1939. Many of the studies were independently significant and, as a group, provided strong evidence of above chance scoring. Likewise, the automated experiments conducted by both Schmidt (1969) and Honorton (1987) produced highly significant results. Also, Honorton and Ferrari (1989) presented a meta-analysis of nearly two million guesses from precognitive forced choice experiments conducted between 1935 and 1987. Although the cumulated effect size was small (0.02), the results were highly significant $\left(\mathrm{p}<10^{-25}\right)$. More recently, Steinkamp, Milton \& Morris (1998) carried out a meta-analysis of 22 forced choice ESP studies that had compared scoring between clairvoyant and precognitive trials. The cumulated outcome of both trial types was highly significant (precognition trials; effect size $=0.01, p=9 \times 10^{-7}$ : clairvoyant trials; effect size $=0.009, \mathrm{p}=.002$ ). The results of many experiments also contain evidence of significant internal effects, with, for example, participants who believe in ESP 
tending to outperform disbelievers (Lawrence, 1993), and trials employing feedback obtaining better results than those giving no feedback (Honorton and Ferrari, 1989).

However, many forced choice ESP studies have been criticised on both methodological and statistical grounds. For example, Hansel (1980) and Gardner (1989) have claimed that some of the early card guessing experiments employed procedures that would have allowed for participant cheating and 'sensory cueing' (i.e., participants inadvertently detecting and utilising subtle signals about the identity of targets). Others have suggested that the highly significant results from some of the automated studies are invalid, as the experiments used nonrandom methods to select and order targets (see, e.g., Hyman, 1981; Kennedy, 1980). Critics have also pointed to possible problems with the way in which data has been collected and analysed. For example, both Leuba (1938) and Greenwood (1938) discussed the potential dangers of 'optional stopping', wherein researchers are able to conclude an experiment when the study outcome conforms to a desired result. Other studies have suffered from the 'stacking effect' - a statistical artefact that can occur when guesses from many participants are all matched against the same target material (see, e.g., Pratt, 1954). The poor levels of methodological and statistical safeguards present in some past studies have been highlighted in two recent meta-analyses. Honorton and Ferrari (1989) analysed the quality of each study in their database by assigning a point for each of 8 major methodological criteria. The studies received an average rating of just 3.3. Likewise, in their meta-analysis, Steinkamp et al (1998) assigned clairvoyant studies a methodological quality rating of between 1 and 19, and reported that the studies obtained a mean rating of just 10.6.

In reply, some parapsychologists have questioned the validity of these criticisms by, for example, arguing that many of the alleged flaws are unlikely to account for reported effects (see, e.g., Palmer, 1986a for a summary), and noted the non-significant correlations between the presence/absence of methodological safeguards and study outcome (see, e.g., Honorton \& Ferrari, 1989; Steinkamp et al, 1998).

The authors aimed to help resolve this debate by devising a novel procedure for carrying out a large scale forced choice ESP experiment. The Mind Machine consisted of a specially designed steel cabinet containing a multi-media computer and large touch screen monitor. The computer presented participants with a series of videoclips that led them through the experiment. During the experiment, participants were asked to complete a forced choice ESP task that involved guessing the outcome of four random electronic coin tosses. All of their data was stored by the computer during an eleven month tour of some of Britain's largest shopping centres, museums and science festivals.

This methodology was developed for several reasons.

First, the study had the potential to collect a huge amount of data from thousands of participants, and thus possess the statistical power to reliably detect the small effect sizes reported in many previous forced choice ESP studies.

Second, it is widely acknowledged that carrying out large scale forced choice ESP experiments is usually problematic, as they tend to be time consuming and tedious for both experimenters and participants alike (Radin, 1997: Broughton, 1991). The Mind Machine overcame these problems by creating a totally automated experiment and by having each participant only contribute a very small number of trials. 
Third, as noted above, many previous forced choice ESP studies have been criticised on various methodological grounds, including sensory shielding, opportunities for participant cheating and poor randomization. The Mind Machine was designed to minimise these potential problems. For example, the computer running the experiment was secured inside a locked cabinet that could not be accessed by participants. Also, possible randomization problems were minimised by having the target selection carried out by a pseudo-random number generator that had been fully tested prior to use.

Fourth, again, as noted above, critics have correctly noted that some previous forced choice ESP studies have suffered from potential statistical problems, including optional stopping and stacking effects. The Mind Machine was designed to overcome these artifacts by specifying the size of the final database in advance of the experiment, and generating a new target sequence for each participant.

Fifth, the Mind Machine methodology could incorporate many of the factors that have positively correlated with study outcome in meta-analyses of previous forced choice studies. Incorporating such 'ESP conducive' procedures was important, given that a previous metaanalysis of forced choice ESP studies conducted via the mass media under non-conducive conditions, had resulted in a cumulative outcome consistent with chance (Milton, 1994). Honorton and Ferrari's (1989) meta-analysis of precognition forced choice studies noted that several factors were significantly associated with increased ESP scoring. Studies providing immediate, trial by trial, feedback to participants obtained significantly higher effect sizes than those giving delayed or no feedback. Also, experiments testing participants individually had significantly higher effect sizes than those employing group testing. Many of these patterns were also found in the meta-analysis carried out by Steinkamp et al. (1998). To maximise the potential of obtaining evidence for ESP, the Mind Machine tested participants individually and provided them with immediate, trial by trial, feedback.

On the basis of the results from previous forced choice ESP studies, it was predicted that participants' overall ESP scores would differ significantly from mean chance expectation.

The Mind Machine also attempted to replicate one of the most reliable internal effects in the forced choice ESP literature. A meta-analysis carried out by Lawrence (1993) revealed that participants who believed in psychic ability tended to obtain significantly higher forced choice ESP scores than disbelievers. To examine this hypothesis, participants in the present experiment were asked to indicate whether they believed in the existence of psychic ability prior to completing the ESP task. On the basis of previous work it was predicted that the ESP scores of believers would be significantly higher than disbelievers' scores.

The study also investigated four other internal effects that have received mixed support in past research.

As noted above, several studies have examined potential differences between ESP scoring on clairvoyant trials (i.e., where the target is selected prior to the participant's choice) and precognition trials (i.e., where the target is chosen after the participants have made their choice). These studies have obtained mixed results. Although a few studies have reported precognition trials outscoring clairvoyance trials (see, e.g., Freeman, 1962; Honorton, 1987), the meta-analytic review conducted by Steinkamp et al. (1998) showed no significant difference between the two types of trials. The Mind Machine followed up on this work by comparing the scoring of clairvoyant trials (where the outcome of half of trials was 
determined prior to participants indicating their guess), with precognition trials (where the outcome of the other half were determined after they had indicated their decision).

Previous forced choice experiments have also examined the potential relationship between ESP scoring and gender. Again, these studies have obtained mixed results, with some reporting females outperforming males, and others showing no significant differences (see, Palmer, 1978 for a review). The Mind Machine experiment examined the potential relationship between gender and ESP scoring.

A small number of past forced choice studies have investigated whether participants' predictions about their performance on a forthcoming ESP task is related to their actual performance. Some of these experiments have shown a significant relationship between predicted and actual performance (see, e.g., Smith, Wiseman, Machin, Harris \& Joiner, 1997: Musso, 1965; Schmeidler, 1971) but others have failed to obtain this effect (see, Palmer, 1978 for a review). Participants in the Mind Machine were asked to predict how many coin tosses they believed they would guess correctly, and the relationship between their predicted and actual success examined.

The experiment also explored whether participants taking part in the study for the first time would obtain significantly different ESP scores to those repeating the experiment. The small number of forced choice studies that have examined participant's ESP performance over several sessions have tended to report a decline effect (see, e.g., Honorton \& Carbone, 1971, Humphrey, 1945). At the start of the Mind Machine experiment participants were asked to indicate whether this was the first time they had taken part in the experiment, and thus it was possible to examine the ESP scoring of 'novices' to participants who had taken part in the study before.

\section{Apparatus}

Hardware

The experiment was carried out on an Omega Science PC with a P200MMX INTEL Processor, Elite TX 512K Motherboard AT, 3.5 Gb hard drive and 16MB-72 Pin EDO Sims. This computer was connected to a 17 inch colour touch sensitive monitor.

Both computer and monitor were housed within a Barcrest steel cabinet specially constructed for high volume public use. This cabinet measured approximately $1.5 \mathrm{~m} \times 0.5 \mathrm{~m} \times 0.5 \mathrm{~m}$ and allowed participants to use the touch screen monitor, but prevented them from having access to the computer and its peripherals (e.g., mouse and keyboard) by securing them in the base of the cabinet. The base of the cabinet could be accessed via panels at the front and rear of the cabinet. Both panels were always locked whenever the cabinet was left in a public venue, and the keys were retained by the experimenters throughout the project.

Each venue provided a continuous $240 \mathrm{v}$ electricity supply. The lead connecting the power supply to the computer was secured in such a way as to prevent it being pulled free from the cabinet. If the supply was turned off, or disconnected at the mains, the computer failed to operate. When the power was reinstated the computer automatically booted into the Mind Machine program.

\section{Software}

The Mind Machine program presented participants with a series of videoclips containing an experimenter (RW) who led them through the experiment. These videoclips were filmed in 
RW's laboratory and were accompanied by background music written especially for the program. Some parapsychologists have emphasised the importance of creating forced choice ESP tasks that participants find both interesting and absorbing. The Mind Machine was able to achieve this by using multi-media videoclips to create an engaging and enjoyable experiment. An evaluation of the program prior to the study revealed that participants understood the purpose of the experiment and ESP task, and found taking part both interesting and engaging. Taking part in the experiment took approximately two and a half minutes, and the engaging nature of the program is reflected in the fact that only $0.85 \%$ of participants did not complete the experiment.

The program initially displayed a videoclip designed to attract participants to the cabinet. This videoclip contained the Mind Machine logo and the phrase 'Touch the screen to begin'. When participants touched the screen, the computer presented a videoclip of RW welcoming them to the experiment and asking them to first answer four simple questions.

The screen was then partitioned into three sections. The top left of the screen displayed short videoclips of RW asking each of the questions. The top right of the screen contained the same question in text form. The bottom half of the screen contained large virtual buttons displaying possible answers to the questions. Participants were first asked whether they were male or female (Possible answers: Male, Female), whether they believed that psychic ability existed (Yes, Uncertain, No), whether it was the first time that they had taken part in the experiment (Yes, No) and how many coin tosses they believed they would guess correctly ( 0 , $1,2,3,4)$.

The program next randomly determined the order of the two clairvoyant (C) and two precognition $(\mathrm{P})$ trials. This was achieved using an inversive congruential pseudo-random number generator (ICG $(2147483647,1,1,0))$ obtained from the P-Lab website (http://random.mat.sbg.ac.at/). This PRNG has been fully evaluated for randomness, and the website contains the results of this extensive testing. The PRNG was seeded from the number of milliseconds that had elapsed since the computer system had started using the Windows 'GetTickCount' API function. For this part of the experiment, the PRNG was programmed to return a number between 1 and 0 . If this value was less than 0.5 then the resulting trial order became P-C-P-C. If the value was greater than, or equal to, 0.5 then the order became C-P-C-P.

Next, the computer randomly determined if the target for clairvoyant trials ('TargetC') would be a 'Head' or a 'Tail'. The PRNG algorithm was programmed to provide an integer between 0 and 99 inclusive, and an even value was coded as a 'Tail' and an odd value as a 'Head'.

The next videoclip showed RW in the centre of the screen, and two large coins in the top left and right of the screen. The coin on the left of the screen displayed a Head whilst the coin on the right displayed a Tail. RW asked participants to touch one of the coins to indicate whether they believed the coin would land Heads or Tails.

The computer recorded the participant's guess and then randomly determined if the target for precognitive trials ('TargetP') was a 'Head' or a 'Tail'. Again, the PRNG was used to provide an integer between 0 and 99 inclusive, and an even value was coded as a 'Tail' and an odd value as a 'Head'. 
For clairvoyant trials the participant's choice was compared to TargetC. For precognitive trials the participant's choice was compared to TargetP. If the participant's choice matched the target then the trial was classified as a 'hit', otherwise it was classified as a 'miss'. A videoclip then informed participants whether they had obtained a hit or a miss, and revealed the outcome of the coin toss. After all four trials had been completed a videoclip informed participants of their total score.

A videoclip then asked participants the percentage of trials they thought that people should get correct by chance alone $(0 \%, 25 \%, 50 \%, 75 \%, 100 \%)$. This question was designed to test the notion that participants who disbelieve in the paranormal will outperform believers on tests of probability estimation (see, e.g., Blackmore \& Troscianko, 1985). The results of this question, along with other psychological data, will be reported in a separate paper.

Finally, a videoclip thanked participants for taking part and presented them with an opportunity to see a final videoclip that would explain more about the experiment. At the end of each session the program returned to the initial videoclip containing the Mind Machine logo. The computer also returned to this initial videoclip from any point in the program if the computer screen remained untouched for 25 seconds.

Participants' data was stored in two databases. The first database was trial based, and contained the participant's number, the trial number, whether the trial was clairvoyant or precognitive, the time of the trial, the value of TargetC, the value of TargetP, whether the participant's choice was a 'Head' or a 'Tail', whether the target was a 'Head' or a 'Tail', whether the trial was a hit or a miss and the number of milliseconds that participant took to make their decision. The second database was participant based, and consisted of the participant's number, the date of the session, their gender, belief in psychic ability, whether it was the first time that they had taken part in the experiment, the number of coin tosses they expected to guess correctly, the time of the first trial, the total number of precognitive trial completed, the total number of hits obtained in precognitive trials, the total number of clairvoyant trials completed, the total number of hits obtained in clairvoyance trials and the percentage of guesses that they believed would be obtained by chance alone. These two databases were regularly backed up throughout the duration of the experiment.

Minimising optional stopping

The Mind Machine experiment could, potentially, suffer from two types of optional stopping.

First, participants' performance on initial ESP trials could influence whether they completed the experiment. For example, participants might obtain two misses on the first two trials, become disappointed and not complete the remaining two trials. Alternatively, participants might perform well on the first two trials and not complete the remaining trials to avoid the possibility of them obtaining misses. To prevent either scenario from creating a potential artefact, data from every ESP trial was included in the database (i.e., participants did not have to complete all four trials to be included in the database).

A second form of 'optional stopping' can occur when experimenters do not set sample sizes in advance of a study, and thus have the potential to terminate the study when the results reach a desired outcome. This potential problem can be avoided by experimenters setting the final sample size in advance of the study, and not examining their data until the experiment has terminated (Milton \& Wiseman, 1997). Before starting the experiment, the authors decided that each of the participants' screen presses would constitute a single datapoint (i.e., 
participants completing the experiment would have provided 9 datapoints - the answers to the initial four questions, the four ESP trials, and probability question) and that the final database would consist of 250,000 datapoints. In addition, the data from the forced choice ESP task was not examined until the end of the study.

\section{The Tour}

The Mind Machine was taken on an eleven month tour of some of Britain's leading museums, science festivals and shopping centres. Before embarking on the tour, and at various intervals during the tour, the Mind Machine was placed on campus at the University of Hertfordshire. The complete list of venues and tour dates is shown in Appendix A.

\section{Results}

The final database contained 27,856 participants providing 250,002 datapoints $(139,043$ datapoints from the five questions and 110,959 ESP trials).

\section{Statistical Power}

The ESP task used in the Mind Machine experiment involved guessing the outcome of a random coin flip. Radin (1997) has estimated that the overall results of past clairvoyant studies would produce an actual hit rate of approximately $54 \%$ on such a task. A power analysis suggested that the current study, containing 110,959 trials, would have a $99.99 \%$ chance of detecting such an effect.

Testing randomness

There exists a great deal of debate around the concept of randomness in parapsychology (see, e.g., Gilmore, 1989, Palmer, 1989). However, for the purposes of ESP testing, most researchers (see, e.g., Palmer, 1986b) consider it important to demonstrate both equiprobability (i.e., each target should be selected an approximately equal number of times) and intertrial independence (i.e., each target has an equal opportunity of occurring after any other target). Each issue will be discussed in turn.

\section{Equiprobability}

Chi-squared analyses revealed that the frequency with which the computer selected Heads and Tails did not differ significantly from chance on any of the four trials, either separately or combined (see Table 1).

\begin{tabular}{|l|l|l|l|l|l|}
\hline & $\begin{array}{l}\text { Number } \\
\text { of Heads }\end{array}$ & $\begin{array}{l}\text { Number } \\
\text { of Tails }\end{array}$ & $\begin{array}{l}\text { \%o of } \\
\text { Heads }\end{array}$ & Chi-square & $\begin{array}{l}\text { p-value } \\
\text { (2 tailed) }\end{array}$ \\
\hline Trial 1 & 13801 & 14055 & 49.54 & 1.14 & .29 \\
\hline Trial 2 & 13818 & 13965 & 49.73 & .38 & .54 \\
\hline Trial 3 & 13934 & 13767 & 50.30 & .48 & .49 \\
\hline Trial 4 & 13839 & 13780 & 50.11 & .06 & .81 \\
\hline Combined & 55392 & 55567 & 49.92 & .14 & .71 \\
\hline
\end{tabular}

Table 1: Number of trials on which the computer selected Heads and Tails, \% of Heads, chi-square values (with continuity correction) and p-values (2-tailed) for each of the four trials separately and combined.

Intertrial dependency

Table 2 contains the results of chi-squares comparing the frequency with which Heads and Tails were chosen between each of the four trials. The analysis comparing Trial 2 and Trial 3 
was significant. Further investigation revealed that this significance was due to the target sequence showing a slight (approximately 1\%) tendency to avoid repeating Trial 2's target on Trial 3 (see Table 3). As the vast majority of participants only completed the experiment once, it is highly unlikely that they would be able to detect and utilise this small bias. However, it is theoretically possible that the effect could coincide with a more general response bias, and thus lead to artefactual findings. To assess this possibility it was decided to analyse the data both including and excluding the only trial which could be effected by the bias, namely, Trial 3.

\begin{tabular}{|l|l|l|l|}
\hline & Trial 1 & Trial 2 & Trial 3 \\
\hline Trial 2 & $.16(.69)$ & & \\
\hline Trial 3 & $.009(.93)$ & $7.91(.005)$ & \\
\hline Trial 4 & $.19(.66)$ & $.04(.83)$ & $.06(.81)$ \\
\hline
\end{tabular}

Table 2: Chi-square values (with continuity correction) and p-values ( 2 tailed, in brackets) comparing the frequency with which targets were chosen between each of the four trials.

\begin{tabular}{|l|l|l|l|l|}
\hline \multicolumn{2}{|c|}{} & \multicolumn{3}{|c|}{ Trial 2 } \\
\cline { 3 - 5 } \multicolumn{2}{|c|}{} & Heads & Tails & Total \\
\hline \multirow{4}{*}{ Trial 3 } & Heads & $48.88 \%$ & $51.12 \%$ & $100 \%$ \\
& & $(6811)$ & $(7123)$ & $(13,934)$ \\
\cline { 3 - 5 } & Tails & $50.58 \%$ & $49.42 \%$ & $100 \%$ \\
& & $(6963)$ & $(6804)$ & $(13,767)$ \\
\cline { 2 - 5 } & Total & $49.72 \%$ & $50.28 \%$ & \\
& & $(13,774)$ & $(13,927)$ & \\
\hline
\end{tabular}

Table 3: The percentage and frequency (in brackets) with which the computer selected Heads and Tails as targets on Trial 2 and Trial 3.

\section{ESP scoring - All data}

Table 4 contains the number of participants, number of hits, number of trials, percentage of hits, z-scores, and p-values ( 2 tailed) for the overall database and each sub-group of data. None of the analyses were significant.

\begin{tabular}{|c|c|c|c|c|c|}
\hline & N hits & $\mathbf{N}$ trials & \% hitting & z-score & p-value \\
\hline \multicolumn{6}{|c|}{ All trials $(\mathrm{N}=27,856)$} \\
\hline & 55,375 & 110,959 & 49.90 & -.62 & .53 \\
\hline \multicolumn{6}{|l|}{ Trial type } \\
\hline Clairvoyance & 27,787 & 55,481 & 50.08 & .39 & .70 \\
\hline Precognition & 27,588 & 55,478 & 49.73 & -1.28 & .20 \\
\hline \multicolumn{6}{|c|}{ Belief in psychic ability } \\
\hline $\begin{array}{l}\text { Believers } \\
(\mathrm{N}=16,150)\end{array}$ & 32,103 & 64,336 & 49.90 & -.51 & .61 \\
\hline $\begin{array}{l}\text { Uncertain } \\
(\mathrm{N}=7476)\end{array}$ & 14,834 & 29,817 & 49.75 & -.86 & .39 \\
\hline $\begin{array}{l}\text { Disbelievers } \\
(\mathrm{N}=4230)\end{array}$ & 8438 & 16,806 & 50.21 & .53 & .60 \\
\hline
\end{tabular}




\begin{tabular}{|c|c|c|c|c|c|}
\hline \multicolumn{6}{|c|}{ Predicted success } \\
\hline & & 12007 & 1077 & & \\
\hline$(\mathrm{N}=3293)$ & 6512 & 13,091 & 49.12 & -.63 & .53 \\
\hline $\begin{array}{l}\text { Chance } \\
(\mathrm{N}=13,630)\end{array}$ & 27,078 & 54,380 & 49.79 & -.96 & .34 \\
\hline $\begin{array}{l}\text { Above chance } \\
(\mathrm{N}=10,933)\end{array}$ & 21,785 & 43,482 & 50.10 & .42 & .67 \\
\hline \multicolumn{6}{|l|}{ First time? } \\
\hline $\begin{array}{l}\text { Yes } \\
(\mathrm{N}=24,665)\end{array}$ & 49,039 & 98,311 & 49.88 & -.74 & .46 \\
\hline $\begin{array}{l}\text { No } \\
(\mathrm{N}=3191)\end{array}$ & 6336 & 12,648 & 50.00 & .20 & .84 \\
\hline
\end{tabular}

Table 4: The number of participants, number of hits, number of trials, percentage of hits, z-scores and p-values ( 2 tailed) for the overall database and each sub-group of data.

\section{ESP scoring - excluding Trial 3}

Table 5 contains the number of hits, number of trials, percentage of hits, z-scores, and pvalues ( 2 tailed) for the overall database and each sub-group of data excluding Trial 3. None of the analyses were significant.

\begin{tabular}{|c|c|c|c|c|c|}
\hline & $\mathbf{N}$ hits & $\mathrm{N}$ trials & \% hitting & z-score & p-value \\
\hline All trials & 41,625 & 83,258 & 49.99 & -.02 & .98 \\
\hline \multicolumn{6}{|l|}{ Trial type } \\
\hline Clairvoyance & 20,881 & 41,656 & 50.13 & .51 & .61 \\
\hline Precognition & 20,744 & 41,602 & 49.86 & -.55 & .58 \\
\hline \multicolumn{6}{|c|}{ Belief in psychic ability } \\
\hline Believers & 24,130 & 48,276 & 49.98 & -.07 & .94 \\
\hline Uncertain & 11,132 & 22,370 & 49.76 & -.70 & .48 \\
\hline Disbelievers & 6363 & 12,612 & 50.45 & 1.01 & .32 \\
\hline \multicolumn{6}{|c|}{ Predicted success } \\
\hline Below chance & 4891 & 9828 & 49.77 & -.45 & .65 \\
\hline Chance & 20,443 & 40,798 & 50.11 & .43 & .67 \\
\hline Above chance & 16,291 & 32,632 & 49.92 & -.27 & .79 \\
\hline \multicolumn{6}{|l|}{ First time? } \\
\hline Yes & 36,890 & 73,762 & 50.01 & .06 & .95 \\
\hline No & 4735 & 9496 & 49.86 & -.26 & .79 \\
\hline
\end{tabular}

Table 5: The number of hits, number of trials, percentage of hits, z-scores and p-values ( 2 tailed) for the overall database and each sub-group of data with Trial 3 excluded.

Table 6 contains the z-scores and p-values ( 2 tailed) for each of the internal comparisons both including and excluding Trial 3. Again, none of the analyses was significant. 


\begin{tabular}{|l|l|l|l|l|}
\hline & \multicolumn{2}{|c|}{ All Data } & \multicolumn{2}{c|}{ Excluding Trial 3 } \\
\hline & z-score & $\begin{array}{l}\text { p-value } \\
\text { (2 tailed) }\end{array}$ & z-score & $\begin{array}{l}\text { p-value } \\
\text { (2 tailed) }\end{array}$ \\
\hline $\begin{array}{l}\text { Trial type: } \\
\text { Clairvoyance vs Precognition }\end{array}$ & 1.18 & .24 & .76 & .45 \\
\hline $\begin{array}{l}\text { Belief in psychic ability: } \\
\text { Believers vs Disbelievers }\end{array}$ & -.70 & .48 & -.93 & .35 \\
\hline $\begin{array}{l}\text { Predicted success: } \\
\text { Below vs above chance }\end{array}$ & -.75 & .45 & -.26 & .79 \\
\hline $\begin{array}{l}\text { First time: } \\
\text { Yes vs no }\end{array}$ & -.44 & .66 & .26 & .79 \\
\hline
\end{tabular}

Table 6: The z-scores and p-values ( 2 tailed) for internal effects both including and excluding Trial 3.

\section{Discussion}

The Mind Machine aimed to obtain evidence for ESP under conditions that minimised the possibility of methodological and statistical flaws, and maximised conditions that previous literature had found to be ESP-conducive. The experiment involved 27,856 participants contributing 110,959 trials, and thus had the statistical power to detect the possible existence of a very small effect. However, the experiment's overall outcome did not differ from chance, and all of the internal analyses were non-significant. These findings could be interpreted in one of two ways.

First, the experiment may have obtained chance results because the conditions under which it was conducted were not conducive to ESP. Despite designing the experiment to incorporate many of the factors that have positively correlated with study outcome in meta-analysis of previous forced-choice ESP experiments, there were several differences between the Mind Machine experiment and most laboratory studies. For example, unlike the vast majority of laboratory studies, participants taking part in the Mind Machine experiment did not have any contact with a 'live' experimenter. Also, the Mind Machine experiment took place in relatively noisy public spaces compared to the quiet surroundings of a laboratory. Finally, the Mind Machine may have sampled from a different participant population to laboratory studies, with participants coming across the experiment whilst visiting a public venue, versus being sufficiently motivated to volunteer to take part in a laboratory experiment (see Milton, 1994).

Alternatively, the study may have failed to find evidence of forced choice ESP because such an effect doesn't exist. Given that the study maximised safeguards against the type of methodological and statistical problems associated with many previous forced choice ESP experiments, the lack of any significant effects would support the notion that the positive findings reported in these previous experiments were spurious.

Assessing these competing explanations is problematic, in part, because it is difficult to determine whether the differences in conditions between the Mind Machine experiment, and laboratory-based research, could account for the results obtained in this study. For example, to our knowledge, only one study has examined the relationship between the degree of participant-experimenter contact and forced choice ESP performance. Steinkamp et al. (1998) rated 22 forced choice ESP studies on three different levels of participantexperimenter contact (direct contact, indirect contact - e.g., mail, and no contact), and 
reported that these ratings were not correlated with study outcome. The notion that ESP performance might be influenced by environmental factors has been more fully examined, but has obtained mixed results (see Palmer, 1978 for a review). For example, Beven (1947) reported that participants carrying out an ESP card guessing task in good light outperformed those in a darkened room. However, Reed (1959) found that different types of background music had no effect on ESP scoring. Finally, no research has compared the ESP scores of the type of participants taking part in public versus laboratory experimentation. Future work could start to tease apart these competing ideas by designing another Mind Machine experiment to examine the relationship between these variables and ESP performance (e.g., by placing the cabinet in locations that differed in levels of environmental distractions etc.).

The Mind Machine methodology can gather a huge amount of data in a relatively short period of time. The way in which this data is collected allows experimenters to test hypotheses that would be problematic to examine in laboratory based research (e.g., the almost continuous stream of data would allow investigators to examine time of day effects, or performance over a very large number of conditions). In addition, the methodology provides access to a more representative cross section of the public than the vast majority of laboratory research. Finally, in line with some of the mass participation studies conducted in the very early days of academic psychology (see, e.g., Perloff \& Perloff, 1977), this type of mass participation study is likely to prove an effective tool for promoting the public understanding of certain research methods. For these reasons, the authors urge researchers to consider adopting this unusual methodology to carry out additional mass participation studies in other areas of psychology and parapsychology.

\section{References}

Blackmore, S. \& Troscianko, T. (1985). Belief in the paranormal: Probability misjudgements, illusory control and the 'chance baseline shift'. British Journal of Psychology, 81, 455-468.

Beven, J. M. (1947). ESP tests in light and darkness. Journal of Parapsychology, 11, 76-89.

Broughton, R. S. (1991). Parapsychology: The Controversial Science. New York: Ballantine Books.

Freeman, J. (1962). An experiment in precognition. Journal of Parapsychology, 26, 123-130.

Gardner, M. (1989). How Not To Test A Psychic. Buffalo, NY: Prometheus Press.

Gilmore, J. B. (1989). Randomness and the Search for Psi. Journal of Parapsychology, 53(4), 309-340.

Greenwood, J. A. (1938). An empirical investigation of some sampling problems. Journal of Parapsychology, 2, 222-230.

Hansel, C. E. M. (1980). ESP and Parapsychology: A Critical Re-evaluation. Buffalo: Prometheus Books.

Honorton, C. (1987). Precognition and real-time ESP performance in a computer task with an exceptional subject. Journal of Parapsychology, 51, 291-320. 
Honorton, C. \& Carbone, M. A. (1971). A preliminary study of feedback-augmented EEG alpha activity and ESP card-guessing performance. Journal of the American Society for Psychical Research, 65, 66-74.

Honorton, C. \& Ferrari, D. C. (1989). 'Fortune telling': A meta-analysis of forced choice precognition experiments, 1935-1987. Journal of Parapsychology, 53, 281-309.

Humphrey, B. M. (1945). Further position effects in the Earlham College series. Journal of Parapsychology, 9, 26-31.

Hyman, R. (1981). Further comments on Schmidt's PK experiments. Skeptical Inquirer, 5(3), 34-40.

Kennedy, J. E. (1980). Learning to use ESP: do the calls match the targets or do the targets match the calls? Journal of the American Society for Psychical Research, 74, 191-209.

Lawrence, T. (1993). Gathering in the sheep and goats...A meta-analysis of forced choice sheep-goat ESP studies, 1947-1993. In Parapsychological Association 36th Annual Convention: Proceedings of Presented Papers, pp 75-86.

Leuba, C. (1938). An experiment to test the role of chance in ESP research. Journal of Parapsychology, 2, 217-221.

Milton, J. (1994). Mass-ESP: A Meta-Analysis of Mass-Media Recruitment ESP Studies. The Parapsycholgical Association 37th Annual Convention: Proceedings of Presented Papers, pp 284-334.

Milton, J. \& Wiseman, R. (1997). Guidelines for extrasensory perception research. Hatfield: University of Hertfordshire Press.

Musso, J.R.(1965). ESP experiments with primary school children. Journal of Parapsychology, 29, 115-121.

Palmer, J. (1978). Extrasensory Perception: Research Findings. In S. Krippner (Ed.) Advances in Parapsychological Research 2: Extrasensory Perception. New York: Plenum Press. Pp 59-244.

Palmer, J. (1986a). The ESP Controversy. In H. L. Edge, R. L. Morris, J. Palmer \& J. H. Rush (Eds.) Foundations of Parapsychology, London: Routledge \& Kegan Paul. Pp 161-183.

Palmer, J. (1986b). Statistical methods in ESP research. In H. L. Edge, R. L. Morris, J. Palmer \& J. H. Rush (Eds.) Foundations of Parapsychology, London: Routledge \& Kegan Paul. Pp 138-160.

Palmer, J. (1989). A Reply to Gilmore, Journal of Parapsychology, 53(4), 341-344.

Perloff, R. and Perloff, L. S. (1977). The Fair - An opportunity for Depicting Psychology and for Conducting Behavioural Research, American Psychologist, 32, 220-229. 
Pratt, J. G., Rhine, J. B., Smith, B. M., Stuart, C. E., \& Greenwood, J. A. (1940/1966). Extrasensory perception after sixty years. Boston: Bruce Humphries.

Pratt, J. G. (1954). The variance for multiple-calling ESP data. Journal of Parapsychology, $18,37-40$.

Radin, D. (1997). The Conscious Universe: The Scientific Truth of Psychic Phenomena. San Francisco: Harper Collins.

Reed, B. (1959). A comparison of clairvoyance test results under three different testing conditions. Journal of Parapsychology, 23, 142 (Abstract).

Schmeidler, G. R. (1971). Mood and attitude on a pretest as predictors of retest ESP performance. Journal of the American Society for Psychical Research, 65, 324-335.

Schmidt, H. (1969). Clairvoyance tests with a machine. Journal of Parapsychology, 33, 300306.

Smith, M., Wiseman, R. J., Machin, D., Harris, P. \& Joiner, R. (1997). Luckiness, Competition, and Performance on a Psi Task. Journal of Parapsychology, 61(1), 33-44.

Steinkamp, F., Milton, J. \& Morris, R. L. (1998). A Meta-analysis of Forced-Choice Experiments Comparing Clairvoyance and Precognition. Journal of Parapsychology, 62, 193-218. 
Appendix A: Venues, location and dates of the Mind Machine tour.

\begin{tabular}{|c|c|c|}
\hline & Dates & Venue \\
\hline 1 & 26 January - 19 March & University of Hertfordshire \\
\hline 2 & 20 March - 21 March & Brent Cross Shopping Centre, London \\
\hline 3 & 22 March - 28 March & University of Hertfordshire \\
\hline 4 & 29 March - 31 March & Olympia Exhibition Centre, London \\
\hline 5 & 1 April - 20 April & $\begin{array}{lll}\text { International } & \text { Science } & \text { Festival, } \\
\text { Edinburgh } & & \\
\end{array}$ \\
\hline 6 & 24 April - 25 April & Fortean Times Unconvention, London \\
\hline 7 & 26 April - 22 June & INSPIRE Science Centre, Norwich \\
\hline 8 & 23 June - 29 June & University of Hertfordshire \\
\hline 9 & 30 June - 4 July & $\begin{array}{l}\text { Earl's Court Exhibition Centre, } \\
\text { London }\end{array}$ \\
\hline 10 & 5 July - 8 August & Lakeside Shopping Centre, Essex \\
\hline 11 & 9 August - 27 August & $\begin{array}{lll}\text { Marlows } & \text { Shopping } & \text { Centre, } \\
\text { Hertfordshire } & & \\
\end{array}$ \\
\hline 12 & 28 August - 11 September & University of Hertfordshire \\
\hline 13 & 13 September- 18 September & $\begin{array}{l}\text { British Association for the } \\
\text { Advancement of Science Annual } \\
\text { Festival, Sheffield }\end{array}$ \\
\hline 14 & 19 September - 13 December & MetroCentre, Gateshead \\
\hline 15 & 14 December - 23 December & University of Hertfordshire \\
\hline
\end{tabular}

